when actually communicated to the system, its protective power continues through life.

4. That therefore, when smallpox prevails in an epidemic form, a general re-raccination is not necessary; for it will give no additional protection to those who have already been duly vaccinated, but is troublesome, attended with some expense, and not wholly free from danger.

5 th. That many of the cases of varioloid after cowpox are attributable to imperfect vaccination, and therefore that this operation should be entrusted to competent and skilful persons only.

March 20th, 1860.

\title{
A SUCCESSFUL CASE OF OVARIOTOMY.
}

BY A. B. CROSBY, A.M., M.D.

[Communicated for the Boston Medical and Surgical Journal.]

IN the town of Franconia, N. H., and near the base of Mount Lafayette, there lived in the summer of 1859-and there still livesa Mrs. S. R., a woman of intelligence, and the wife of a machinist, formerly of Providence, R. I. This lady consulted my father, Prof. Dixi Crosby, in the month of May, with regard to an ovarian tumor, of which she was the victim. After a critical examination, he advised her to submit to ovariotomy, and with a generositycreditable to a parent, but unusual in a surgeon-he relinquished the case into my hands.

On the night previous to the operation, Aug. 27th, I derived from the patient the following history of her case. She was 36 years of age, and had been married eleven years. Two children were the fruit of this connection-a girl and a boy, respectively, ten and eight years of age. The father of the patient died of general dropsy at the age of 77. She lost her paternal grand. mother at the age of 50, and an aunt at 60-both from the same disease. The patient menstruated at 14 . At 16, she paddled with her feet in the cold water of a brook, and there was no catamenial discharge for a year afterwards. Aside from this, her health was good. She married at 25 , and for six months previous had scanty, high-colored urine, and painful micturition. The first child was born in eleven months after marriage. The labor lasted sixteen hours, and was natural. In eighteen months after the first labor, the second child was born at eight months. Four weeks previous she slipped, and came near falling, by which she strained her back. The second labor occupied four hours and a half, and was natural. Menstruation has been regular ever since, recurring as often as every third week. The general health of the patient has been excellent during most of the time.

One night in February, 1854, she discovered a tumor, about the size of a small hen's egg, over the right ovary, and "played with it 
some time." In the course of three months, the tumor became as large as "both fists." It began to be somewhat tender, and she consequently let it alone. At the end of six months, it had become a large symmetrical tumor, confined to the right side.

From the fact that she was troubled with nausea and vomiting, the patient supposed herself pregnant, although menstruation was regular during the whole period. At the commencement of the second year, she became debilitated and exhausted.

After the tumor had existed twenty-eight months, Dr. Bugby, of Littleton, N. H., tapped the patient, and drew away twenty-six and a half pounds of a perfectly limpid fluid, slightly glutinous. Great prostration ensued, and she was confined to her bed for a fortnight. At the expiration of thirteen wecks after the first tapping, Dr. Bugby removed from the cyst thirty and a half pounds of a fluid resembling almost preciscly that of the former tapping.

In eight weeks the tumor was again as large as crer. She now took diuretics freely, and in the course of two or three days a profuse diuresis occurred, which reduced the size of the tumor one half. Fifteen months now elapsed, at the end of which time Dr. D. E. Wells, of Franconia, drew away thirty-five pounds of a limpid, viscid fluid, resembling the white of an egg. Dr. Wells tapped the patient, in all, twelve times, at regular intervals of three weeks and a half. The interval between the last two tappings, however, was only a week and a half. At the last two operations only one half of the contents of the tumor was removed. The average quantity removed each time was thirty-five pounds. The fluid gradually became thicker, until towards the last it rescmbled fluid starch in color and consistence. Paracentesis was performed for the last time, August 16th.

At the time of the final opcration, the tumor presented, externally, a perfectly symmetrical appearance. The abdominal wall was much attenuated, and so far distended that the patient could not see her feet when in the erect position. On applying one hand to the side of the tumor and communicating a sharp tap to the opposite side, a distinct wave could be felt. She had been troubled with umbilical hernia, but the abdominal wall could be raised at several points, and caused to slide over the surface of the tumor. The only pain which had occurred during the disease was of comparatively recent date.

The fearful risk of the operation, and the probabilities of a fatal issue in the case, were fully represented. But her courage was indomitable, and she met the issue with great firmness and checr. fulness. Her genuine old Saxon pluck under the impending danger excited the undisguised admiration of all who witnessed it.

The bowels having been thoroughly evacuated by castor oil, and some lines drawn across with nitrate of silver at right angles to the linea alba, to serve as a guide for the sutures, the operation was performed on Sunday morning, Aug. 28th, at $9 \frac{1}{2}$ o'clock, A.M. 
There were present, Prof. D. Crosby, Dr. Bugby, Senior, of Waterford, Vt., Dr. Bugby, Junior, of Littleton, N. H., Dr. Baynton, of Lisbon, N. H., and the attending physician, Dr. Wells, of Franconia.

The tempcrature of the room was brought up to eighty degrees, and maintained there during the operation. An abundance of steam was generated from shallow basins on the stove, in order that the peritoneum might not suffer from dryness. The white of an egg, diluted with water, and moderately warm, was prepared for the fingers and instruments which were to come in contact with this delicate membrane.

Everything being prepared, Mrs. R. went upon the table with a smile, and indicated her perfect composure by a steady pulse of $\mathbf{7 2}$. Chloroform was administered by iny father, the patient coming under its influence quictly and without excitement. Dr. Wells on one side, and Dr. Bugby, Jr., on the other, by a firm, but gentle pressure of the hands, supported the abdomen. I commenced the operation by making an incision four inches in length, extending from the left side of the umbilicus through the linea alba to the pubes. This incision extended as far as the peritoneum. A small opening having been made through that membrane, the patient commenced vomiting, and I was obliged to discontinue the use of the knife. In four or five minutes, the stomach having become quiet, I passed a male catheter between the abdominal wall and the surface of the tumor, in order to swreep the whole anterior surface, and thus aseertain how far adhesions existed. There was a firm connection at the umbilicus, which it required considerable force to separate-an omental attachment, which was broken up by torsion-and a slight adhesion to the diapluragm, which the beak of the catheter separated.

I now divided the peritoneum throughout the whole extent of the wound, and passed a sail needle, armed with a doubled thread, through a fold of the anterior wall of the cyst, in order that I might thereby make tension in effecting its removal. The wall, however, proved very thin, and the loop tearing out, the contents of the tumor began to escape. Directing the assistants on either side to maintain their pressure, I seized the cyst with my fingers and withdrew it as far as possible, directing the flood over the right side of the patient on to the table and floor. About three gallons of an extremely glairy, tenacious fluid escaped in this way. None of it, however, found its way into the abdominal cavity, and I now renewed my attempts to dislocate the tumor.

On introducing my hand, I could feel a symmetrical tumor over the fundus of the uterus, apparently immovable. Firm tension by the cyst seemed to have no effect upon it. At length, requesting my father to introduce his finger under the right extremity of the .tumor, using it as a lever, I seized the cyst with both hands, and by our combined efforts we succeeded in dislodging the solid part 
of the tumor, which had been thus firmly located beneath the promontory of the sacrum.

I now found that the tumor was attached to the right orary, and involved both the broad and round ligaments. With my nail, I divided the round ligament, removing a portion with the tumor, and by the same means separated the broad ligament until the nutrient portion of the pedicle of the tumor was reduced in width to about an inch and a half. I then elevated the tumor as far as possible with both hands, and my father passed a needle, armed with a double ligature, through the centre of the pedicle, and tied firmly both ways. I then surrounded the whole pedicle with a very strong ligature, and released the tumor from its attachment by the knife.

I now introduced bits of old soft linen into the abdomen until they ceased to be stained; then bringing the edges of the wound together, I introduced three silver sutures as far as, but not involving the peritoneum. A twisted suture was employed at the lower angle of the wound, to which the ligatures were firmly tied. A soft compress was placed over the wound, and retained by a swathe passing around the body. The operation occupied about thirty-five minutes, and in an hour from the time the patient was placed on the table she was removed to her bed. The skin was moderately moist throughout, and at the conclusion of the operation the pulse was only 80 -in fact, there was scarcely any appreciable shock.

The patient took a quarter of a grain of morphia, and subsequently took one-eighth of a grain every two hours until night. She vomited several times during the afternoon, and her pulse gradually came up to about 100 . By placing the hands firmly but gently on the bowels, the vomiting did not disturb the wound, and this precaution was taken whenever there was any sign of retching afterwards.

6 o'clock, P.M., patient vomited. 8 o'clock.-Took morphia. 84 o'clock.-Vomited; pulse 104. Finding that the pulse was increasing in frequency, I determined to resort to the veratrum viride, in the hope that by quieting the heart's action I might obviate the tendency to peritonitis.

9 $\frac{1}{2}$ o'clock.-Patient took three drops of the veratrum; a gill of water was drawn by the catheter; pulse 108. 10 o'clock.Sleeping. 11 o'clock.-Took morphia; complained of pain. 113

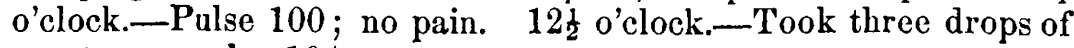
veratrum; pulse 104 .

Aug. 29th, 2 o'clock, A.M.-Took morphia; has been awake for the last half hour, but is free from pain. $2 \frac{1}{2}$ o'clock.-Pulse 96 . $3 \frac{1}{2}$ o'clock.-Took three drops of veratrum; pulse 100. 5 o'clock.

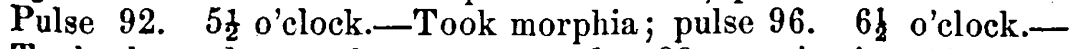
Took three drops of veratrum; pulse 88 ; respiration 12 ; com: plains of no pain. 8 o'clock.-Pulse 88 ; no pain; took morphia. 


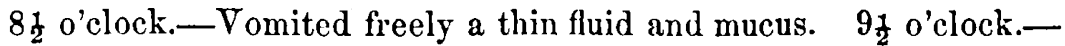
Took three drops of veratrum; pulse 82. 11 o'clock.-Pulse 84; no pain; vomited bilious matter; pupil contracted. $11_{2}^{1}$ o'clock. - Gave an injection of twenty-five drops of laudanum in a little starch.

1 o'clock, P.M.-Took two drops of veratrum; slight pain; pulse 80. $1^{1}$ n'rlock.-Vomited bilious matter; pulse 84. 2 o'clock.-Took morphia; pulse 80 ; pain in the region of the stomach. 3 o'clock.-Gave laudanum injection; pulse 80 ; pain in epigastrium. $\quad 4_{2}^{1}$ o'clock.-Took two drops of veratrum; pulse 84 . 5 o'clock.-Had bilious vomiting and eructations, with relief. Took morphia. $7 \frac{1}{2}$ o'clock.-Took four drops of veratrum; gave laudanum injection; pulse 84. Pain and oppression about the stomach.

Patient has slept at intervals during the day-and has been thirsty. Vomiting has seemed to afford relief. There is no swelling nor tenderness of the bowels. The wound is dry and clean, and the sutures show no signs of irritation. Patient has had no pain whatever in the region of the wound during the day. The urine has been drawn by the catheter four times since the operation, and the quantity is larger than before. The pupil has been contracted during the whole day, and the skin moderately warm. The temperature of the room has been maintained at from $70^{\circ}$ to $75^{\circ}$. She slept most of the time, until midnight, when more urine passed, and a laudanum injection was given. Pulse 84 .

Aug. 30th, $1_{4}^{3}$ o'clock, A.M.-Patient vomited freely; pulse 80. $2 \frac{1}{2}$ o'clock.-Sleeping; pulse 84 ; respiration 10 . $3_{2}^{\mathrm{l}}$ o'clock.Vomited. 4 o'clock.-Has been sleeping; gave laudanum injection; pulse 78. 6 o'clock.-Pulse 80 ; skin natural ; respiration 12.

This morning I was obliged to return home, a distance of some sixty miles; but a careful journal was kept of the case by Dr. Wells, assisted by the patient's husband, from which I have con. densed the following report:-During the remainder of the day until midnight, an injection of starch, containing from fifteen to twenty drops of laudanum, and from two to three drops of veratrum, was administered every three hours. The frequency of the pulse was noted every hour. It was at one period of the day only 70 , the arerage being about 76 . Once only, on suddenly awakening from a frightful dream, the pulse indicated 84, which was its maximum for the day.

During the early part of the day the number of respirations per minute was 10 ; the maximum 15 , and the average 12 . The skin was moderately warm, and the urine was drawn several times by the catheter. A dark menstrual fluid showed itself at intervals, attended by considerable pain in the loins and hips. The patient slept more or less, and at night was treated to a hot sponge bath.

Aug. 31st.-During the first half of the day, the pulse was steady at 72 ; it came up to 84 in the afternoon, and at midnight it was 
100. The injections of starch, laudanum and reratrum were exhibited every four hours. The patient slept the greater part of the time. There was much less thirst after the medicines were in. troduced by the rectum, and no vomiting until this afternoon. She took a little beef tea in the morning, but, in the after part of the day, vomited several times. The bowels also became tympanitic, and she experienced pain in the stomach, bowels, and back. Everything introduced into the stomach began to occasion distress.

Sept. 1st.-I returned to my patient again last night, and found her somewhat depressed. During this day the pulse ranged from 96 to 100 . The respiration was from 12 to 16 . Injections were resorted to as before, every four hours. The patient bore firm pressure on the bowels, although they were tympanitic. She suffered comparatively little pain, and healthy pus discharged around the ligatures. She took small quantities of beef tea through the day, although the stomach was irritable, and vomiting frequent.

Sept. 2d.-Mrs. R. had a pulse to-day ranging from 92 to 100 . Her respiration was most of the time at 12, although occasionally dropping below that number. Four injections were given during the first sixteen hours of the day, the quantity of laudanum and veratrum being gradually diminished. The last night was a restless one, the bowels being swollen, tympanitic and painful. Nausea and vomiting were of frequent occurrence. At 6 o'clock, P.M., the cxtremities suddenly became chilly, and the skin cool and clammy. Severe pain ensued, excessive vomiting occurred, and the patient for the first time expressed her belief that she should die. The pulse was feeble, and the whole system much prostrated. The laudanum and veratrum were of course abandoned. A hot rock was placed at her feet, friction used about the limbs, and carbonate of ammonia and whiskey given by the mouth. She vomited, however, almost incessantly, and complained of great distress at the stomach. After repeated efforts to exhibit stimulants by the stomach, injections of beef tea and carbonate of ammonia were resorted to, with good results.

Sept. 3d.-Until one o'clock this A.M., the distress of the patient from nausea and vomiting continued unabated. From this time until ten o'clock, A.M., the patient was permitted to take absolutely nothing into her stomach. The abdomen was tympanitic; tender and painful. The patient was restless and anxious.

With the assistance of Dr. Wells, I prepared an injection consisting of the tinctures of assafoetida and valerian with the oil of turpentine, using molasses as a vehicle. This soon passed off, bringing away a great amount of flatus and a small quantity of focal matter. At ten o'clock, A.M., we commenced giving a few drops of whiskey with beef tea every hour. During the day the movement of flatus would occasionally give rise to sharp pain. There was also some pain in the vicinity of the wound. As there were borborygmi, together with pain and distension of the bow- 
els at night, an injection similar to that of the morning was exhibited. This brought away a large amount of flatus, with relief, and the patient passed a comfortable night, sleeping at one time two hours. During the latter part of this day the pulse ranged from 92 to 100 , the respiration was 16 , and the tongue covered with a yellowish fur.

Sept. 4th.-At six o'clock, A.M., Mrs. R.'s pulse was 83 ; respiration 20 , skin warm, and the tongue moderately coated. The wound was looking well, and healthy pus escaped from around the ligatures. The fulness of the bowels was much reduced, and the tenderness had almost wholly subsided. Three days ago I removed the twisted suture at the lower angle of the wound, and found that immediate union had taken place. She continued quite comfortable through the day, taking stimulants and beef tea. She was occasionally troubled with colic-pains, but felt, on the whole, quite bright.

Sept. 5th.-I left my patient this morning and returned home, but her condition was as follows:-The pulse ranged from 86 to 96 -and respiration was steady at 16 . She took chicken broth, with other simple nourishment. She expressed a desire for food. The wound discharged healthy pus, and she slept at intervals. Occasionally there were sharp pains in the region of the wound.

Sept. 6th.-To-day the pulse came up to 104 -the respiration to 22 , and the patient showed a disposition to sink. The bowels again became tympanitic, and there was anorexia. Sulphate of quinine was exhibited in grain doses, together with a little whiskey. An antispasmodic injection was employed with some relief. A small dose of castor oil was given, which operated moderately. The night was restless, and painful.

Sept. 10th.-I returned to Franconia to-day, and found Mrs. R. with a pulse of 112 , and feeling feeble. I remained with her three days, and gave her quinine and whiskey freely. Rich broths, oysters, and finally broiled chicken and steak, were given at short intervals. On the 8 th, I removed the silver sutures, which had remained without irritation eleven days, and found that the wound was thoroughly united.

On the 9 th, there were still symptoms of depression, the pulse and respiration being a good deal accelerated. In moving the ligatures a considerable quantity of pus escaped, as though it might have been pent up. Thinking the signs of depression might be owing to an accumulation in the peritoneal cavity, I supported the abdominal wall with both hands, and directed the attendants to turn the patient on her side. A little pus escaped at first, and then about two quarts of serum flowed out by the ligatures. The pulse and respiration diminished in frequency, and the appetite returned almost immediately. Stimulants, quinine and a generous diet were freely resorted to.

On the morning of the 10th, before leaving my patient for the LXII. $-9 * *$ 
last time, I made gentle tension on the ligatures, and brought them all away. This was the fourteenth day after the operation.

On the next day she was put into a chair without inconvenience, and continued to get up daily ever afterwards. Her only subsequent annoyance was from colic pains.

Five weeks after the operation, Mrs. R. was able to dress herself and direct her household affairs. At the end of seven weeks she was able to do her own work and ride three miles to church.

Under date of Jan. 25th, I received a letter from Mrs. R.'s husband, from which I make the following extracts:-

"My wife appears to be as well as she ever was, and I think is as heavy as ever. There has been no menstruation since the dark muddy fluid which you saw just after the operation. She feels well, and eats well."

It remains that I should speak briefly of the tumor. The great bulk of it consisted of a single cyst. The more solid portion was made up of several minute vesicles, which would doubtless have developed, had the disease continued, and have rendered the tumor distinctly multilocular in character. The solid portion weighed a little more than three pounds, and the glairy contents a little less than twenty-five pounds; thus making the weight of the whole mass removed, twenty-eight pounds.

A few considerations, and I have done. The disease of the ovary in this case had existed something more than five years, during which time the cyst was making a constant drain on the fluids of the body. During the period indicated alsore, Mrs. R. lost at the hands of the surgeon about four hundred and seventy-five pounds of fluid. Yet notwithstanding this, her general health is reported as having been good most of the time during the existence of the disease.

It will be remembered that at one time, under the influence of diuretics, a profuse diuresis occurred, and the tumor was diminished one half in size. The generally received opinion has been that an accumulation of fluid in an ovarian cyst is independent of the action of diuretics. My belief is, that in this case a slight rupture of the cyst occurred, and part of its contents escaped into the cavity of the peritoneum. A small obscure cicatrix on the wall of the cyst would seem to corroborate this riew.

Finally, the persistent vomiting in this case merits a passing notice. It was excessive during the first week, and the amount of fluid ejected from the stomach, very greatly exceeded the amount swallowed. No doubt the chloroform, the opium, and the veratrum might have given rise to vomiting, but not, I think, to the extent which existed in this case. My rationale would be this:-For five years the patient's system had been habituated to a constant drain daily. Now after the operation the quantity of urine was only slightly increased, nor was there any unusual excretion either from the bowels or skin. Under these circumstances, may we not sup- 
pose that the vomiting of this large amount of fluid was not an effort on the part of nature to compensate for the sudden suppression of a long-established drain?

In closing this report, I should do myself injustice did I not take occasion to express my thanks to the medical gentlemen who were present and assisted at the operation-and especially to Dr. Wells, who so matcrially aided me in carrying out the details of Mrs. R.'s case.

Hanover, N. H., March, 1860.

\section{RESEARCHES UPON THE ERECTILE ORGANS OF THE FEMALE.}

[Translated for the Bos. Med. and Surg. Journal, by Wu. RrAD, M.D.-Continued from p. 168.]

TaE type of the apparatus essential for generation, in the vertebrata, situated at the lowest step of an ascending scale, that is to say among fishes, presents two varieties, which are in some sort the repetition of what we observe in the invertebrata. The eggs of the myxines, the lampreys, and most species of the murenoids and all the salmonides, detach themselves from the ovarian mass and fall into a cavity (peritoneal) lined with a vibratile epithelium, and communicating externally by exclusive openings (peritoneal canals). The analogy is evident with what happens in the majority of the radiata (polypi, medusæ), and among bryozoons and acephalous mollusks, \&c., in whom the eggs fall from the ovary into a cavity (cavity of the body, bronchial cavity) which communicates freely with the exterior.

The majority of the bony fishes, however, have their generative system constructed after a type analogous to what we observe in the articulata. This type, in the more highly organized species, is common to all those systems which elaborate a product intended to be transmitted externally. The eggs develope themselves upon the parietes, more or less complex, of a special cavity having the form of a sac or a funnel-shaped tube, and opening itself in the most direct course externally. A more or less complete muscular envelope is attached to the glandular apparatus constructed after this type; the generative system of fishes forms no exception to this rule, and it has been now for a long time known that the ovary of fishes had a muscular envelope which sent off into the interior of that organ partitions of the same nature. See Stannius, Anat. Comp., 2, 138.-Leydig (Lehrbuch der Hystologie, 508) has observed muscular fibres penetrating the envelope and the stroma of the ovary in the species Esox, Perca and Salmo. My own investigations have led me to the same results in the lamprey, the tench, aud many species of squales, \&c.

This fact, of the highest importance in the history of this function, has remained entirely neglected, and no one has attempted to connect it with what we observe in the other vertebrata. In my 